\title{
A Morphological Multiphase Active CONTOUR FOR VASCULAR SEgMENTATION
}

\author{
Victoria L. Fox ${ }^{1}$, Mariofana Milanova ${ }^{2}$, and Salim Al-Ali ${ }^{3}$ \\ ${ }^{1}$ Department of Applied Science, University of Arkansas at Little Rock, USA \\ ${ }^{2}$ Department of Computer Science, University of Arkansas at Little Rock, USA \\ ${ }^{2}$ Department of Computer Science, University of Arkansas at Little Rock, USA
}

\begin{abstract}
This paper presents a morphological active contour ideal for vascular segmentation in biomedical images. The unenhanced images of vessels and background are successfully segmented using a two-step morphological active contour based upon Chan and Vese's Active Contour without Edges. Using dilation and erosion as an approximation of curve evolution, the contour provides an efficient, simple, and robust alternative to solving partial differential equations used by traditional level-set Active Contour models. The proposed method is demonstrated with segmented data set images and compared to results garnered from multiphase Active Contour without Edges, morphological watershed, and Fuzzy C-means segmentations.
\end{abstract}

\section{KEYWORDS}

Active Contour, Morphology, Segmentation, Curve Evolution

\section{INTRODUCTION}

Image segmentation is responsible for partitioning an image into sub-regions based on a desired feature and is an essential first task in many disciplines. Biomedical segmentation separates a medical image into different regions based upon pathology, anatomical structure, tissue classes, or many other inherent criteria. Often, these partitions are challenging to construct due to noise, low contrast, and image artefacts embedded in the figure. Methods for biomedical segmentation range from basic thresholding techniques [1],fuzzy logic approaches[2], to intricate partial differential equation models[3].

\subsection{Segmentation Techniques}

Using the assumption that regions of interest in an image are identifiable by separating intensity values, thresholding sets a value in which pixels above the threshold are grouped as the region of interest and pixels below are segmented as background pixels. For images with sharp edges, the method proves effective; once influenced by speckle or varying intensity levels, this approach loses its effectiveness. Region growing techniques build on the idea of thresholding by starting with a seed pixel known to be inside the region of interest. Using a threshold, the neighbourhood of the seed pixel is categorized as foreground or background. This process then performs a search through the pixels of the image classifying each. However, it is difficult to set a threshold which completely confines the region of interest and image leakage is a common shortcoming of the method [4]. 
Fuzzy logic approaches have the advantage of allowing a pixel to belong to multiple clusters in the segmentation. To determine the clusters in which to assign a pixel, the algorithm sets a degree of belonging to each cluster for the pixel. Using this reasoning, a pixel on the edge of the region of interest will have a lower degree of belonging to the cluster than a pixel located near the center of the region of interest. The flexibility inherent in the method involves a trade-off in increased computational complexity. Additionally, noisy images cause a decrease in accuracy of the method [5] due to the nature of its clustering methodology. However, because of its flexibility, a popular fuzzy model - the Fuzzy C-means method - is widely used in segmentation of medical images.

Segmentation methods based on minimization of energy functionals are commonly referred to as active contour methods and are popular due to their ability to always produce sub regions with continuous boundaries. The original active contour method, the snake, and its variations [6 -11] are disposed to large error results when dealing with "false" edges and noisy images. Several implementations, such as the minimal path technique by Cohen et al. [12-13] or dual snakes [14], and other similar methods [15-19], have been suggested to correct the error associated with challenging images. Unfortunately, all of these classical snakes and active contour models can only detect objects with edges defined by the gradient, and, as expected, the performance of the totally edge based methods is often inadequate.

In the past two decades, the creation of a region-based functional that is less likely to give unwanted local minima when compared to the simpler, edge-based energy functions has been an area of active research. The region-based models [20], use information not only near the active contour, but image statistics both inside and outside the contour. In 2001, Chan and Vese [21], based a region-based functional on the Mumford-Shah functional to propose an active contour without edges. For the Active Contours without Edges, the functional of a curve $\mathcal{C}$ is

$$
\begin{aligned}
& F\left(c_{1}, c_{2}, \mathcal{C}\right)=\mu \cdot \operatorname{length}(\mathcal{C})+v \cdot \operatorname{area}(\operatorname{inside}(\mathcal{C}))+ \\
& \quad \lambda_{1} \int_{\text {inside } \mathcal{C}}\left\|I(\boldsymbol{x})-c_{1}\right\| d \boldsymbol{x}+\lambda_{2} \int_{\text {outside } \mathcal{C}}\left\|I(x)-c_{2}\right\| d x
\end{aligned}
$$

where the non-negative parameters $\mu, v, \lambda_{1}, \lambda_{2}$ control the strength of each term and $c_{1}, c_{2}$ provide the statistics of the interior and exterior regions of the contour, respectively.The energy in the Chan-Vese model can be seen as a particular case of the minimal partition problem, and the active contour is evolved in the level set formulation. With the introduction of the Chan-Vese model, region-based models could now handle objects with boundaries not necessarily gradientdefined. However, the computations for the pixel intensities within each region had a high computational cost. Many variations, such as [22] in which the simplicity of the $k$-means algorithm is utilized or [23] in which the algorithm directly calculates the energy alterations rather than solving the underlying PDE equations, have been proposed to improve the efficiency and accuracy of the Chan-Vese model.

\subsection{Morphological Active Contours}

Morphological approaches for image processing include operators for denoising, enhancing, and simplification [24]. In the setting of segmentation, morphology has played a direct role in the evolution of a discrete scheme for the mean curvature motion of level sets [25]. In edge based active contour methods, the contour is composed of three components: a balloon force, a smoothing force, and an edge attraction force. Region based models also contain a balloon force and a smoothing force. Since such models take into account the statistics of the interior and exterior regions of the contour, there is a need to replace the edge attraction force with an image attachment term which provides the statistics needed for the formulation. 
Recent studies in the effectiveness of using morphological operators to drive curve evolution focus primarily on the development of a sequence of erosions and dilations with specific structuring elements. In Jalba and Roerdink's research [26], the authors detail a discrete approach to curve evolution. By iteratively eroding an input set embedding the initial curve by a morphological structuring element, the authors partially segmented a $2 \mathrm{D}$ vascular image and successfully segmented the bones of the human feet in a CT scan. The partial segmentation of the $2 \mathrm{D}$ image is explained as a result of sacrificing accuracy for efficiency.

For Alarez et al., the study [27] is concerned with a full morphological scheme which approximates the action of the Geodesic Active Contour model curve evolution. The scheme is further extended in [25] to an approximation of Active Contours without Edges and turbo pixels. The morphological aspects of the scheme occur in the approximation of the balloon force and smoothing force for the Geodesic Active Contour model and primarily in the smoothing force in the Active Contour without Edges Model. As the morphological equivalent of the mean curvature motion, the smoothing force is comprised of a series linear structuring elements iteratively applied to the contour.

In our approach, we also use a structuring element iteratively applied to the contour to approximate the mean curvature motion of a level-set active contour. Our structuring element is more straightforward in implementation than the series of linear structuring elements applied in [25] or [27] and we achieve more accurate results than Jalba and Roerdink's 2D segmentation. The implementation of the scheme is efficient and robust to noise, blurred edges, and image artefacts in the medical images and easily be extended into three-dimensional applications. Finally, it relies upon region based statistics and can be fully automated in segmentation applications.

\section{A Morphological ACtive Contour Formulation}

The underlying principle of mean curvature motion is the evolution of a simple closed curve whose points move in the direction of the normal with specified velocity [28].

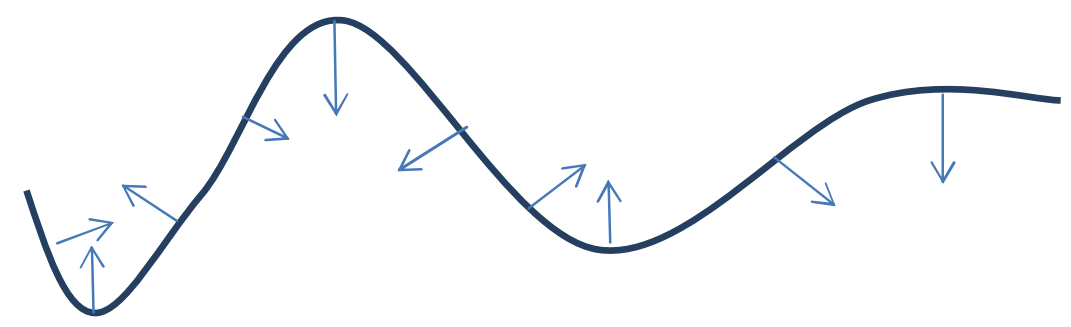

Figure 1: Motion of a curve by curvature. The arrows represent the velocity at some points. Here, the velocity is a nondecreasing function of the curvature.

In the level set framework, $\mathcal{C}$ is implicitly represented by a higher dimensional Lipschtiz function $\phi$ where $\mathcal{C}=\{(x, y) \mid \phi(x, y)=0\}$. The deforming curve is given by the zero level set at time $t$ of function $\phi(x, y, t)$. Evolving the curve in its normal direction with speed $F$ can be achieved by solving

$$
\frac{\partial \phi}{\partial t}=F\|\nabla \phi\|
$$

with the initial condition of $\phi(x, y, 0)=\phi_{0}(x, y)$, where $\phi_{0}(x, y)$ is the initial signed distance function of $\mathcal{C}$. For the Active Contour without Edges functional (1), the steepest descent method gives us this variation of (2): 
International Journal on Bioinformatics \& Biosciences (IJBB) Vol.3, No.3, September 2013

$$
\frac{\partial \phi}{\partial t}=\left\{\mu \cdot \kappa-v+\lambda\left[\left(I-c_{2}\right)^{2}-\left(I-c_{1}\right)^{2}\right]\right\}\|\nabla \phi\| .
$$

In solving for $\phi$, it is important to note that $\kappa$ represents the level set curvature and $c_{1}=$ average $(I)$ in $\{\phi \geq 0\}$ and $c_{2}=$ average $(I)$ in $\{\phi<0\}$. The first term of $F$ in (3) represents the curvature flow and minimizes the curve length; the second term represents inward motion at a constant speed and minimizes the region area; while the last term represents region competition through the statistics of each region.

Focusing on the curvature flow, $F_{\mathrm{K}}=\mu \kappa$, in [28] it is shown that iterating $k$ times a median filter using a window of size $\varepsilon$ converges when $\varepsilon \rightarrow 0, k \rightarrow \infty, \varepsilon k \rightarrow t$ to the mean curvature flow. Additionally, it has also been shown that a median filter can be approximated by a binary morphological opening-closing filter [30] where the structuring element of the opening-closing filter is roughly half the size of the structuring element of the median filter. The structuring element, $\mathcal{B}$, in [28] is the representation of the unit ball created by the Euclidean norm $\|\cdot\|$. Letting $\mathcal{B}_{d}$ represent the discrete version of this structuring element in two-dimensional applications, the authors of this paper chose to use a square structuring element of size $d$ as the closest discrete representation of the continuous case.Therefore, the iterative morphological curvature flow can be represented by

$$
\phi_{k+1}=\left(\phi_{k} \circ \mathcal{B}_{d}\right) \cdot \mathcal{B}_{d},
$$

where ' $\circ$ ' denotes set opening and ' $\bullet$ ' denotes set closing.

Moving to the second term in (3), inward motion at a constant speed, $F_{c}=-v$, with $v$ nonnegative, a weak solution for the PDE describing inwards curve motion at constant speed can be given by eroding the embedded curve $k$ times [26, 28, 31]. Thus, the iterative morphological constant speed for inwards curve motion can be described by

$$
\phi_{k+1}=\phi_{k} \ominus k \mathcal{B}_{d}
$$

where ' $\ominus$ ' represents morphological erosion. Likewise, the iterative morphological constant speed for outwards curve motion can be approximated by dilating the embedded curve $k$ times and can be represented by

$$
\phi_{k+1}=\phi_{k} \oplus k \mathcal{B}_{d}
$$

Finally, in [32] the Heaviside function was introduced to improve the Active Contours without Edges for multiphase segmentation. Due to the nature of the vascular images this paper references, the proposed method incorporates the Heaviside function in its formulation of the region competition portion of (3). Specifically, the Heaviside function can be expressed as

$$
H(\phi)=\left\{\begin{array}{l}
1, \text { if } \phi \geq 0 \\
0, \text { if } \phi<0
\end{array}\right.
$$

and the region competition portion of (1) becomes

$$
\lambda_{1} \int_{\text {inside }}\left\|I(\boldsymbol{x})-c_{1}\right\| H(\phi) d \boldsymbol{x}+\lambda_{2} \int_{\text {outside }}\left\|I(x)-c_{2}\right\|(1-H(\phi)) d x .
$$

The computations $c_{1}$ and $c_{2}$ are relatively straight forward and do not contain much computational complexity. It is obvious that

$$
c_{1}=\int_{\Omega} I \cdot H(\phi) d x / \int_{\Omega} H(\phi) d x^{c_{2}}=\int_{\Omega} I \cdot(1-H(\phi)) d x / \int_{\Omega}(1-H(\phi)) d x
$$


and the proposed method uses the approximations of (9) to help deriving the morphological approximation of the region competition term in (3). Fundamentally, when

$$
\lambda_{2}|\nabla \phi|\left(I-c_{2}\right)^{2}<\lambda_{1}|\nabla \phi|\left(I-c_{1}\right)^{2} \text { at } \boldsymbol{x}
$$

$\boldsymbol{x}$ belongs to the exterior of the contour. When the inequality is reversed, $\boldsymbol{x}$ belongs to the interior. If the inequality becomes an equality, e.g. $\lambda_{2}|\nabla \phi|\left(I-c_{2}\right)^{2}=\lambda_{1}|\nabla \phi|\left(I-c_{1}\right)^{2}$, then $x$ is located on the contour.

With these three terms defined, the algorithm for the proposed method can be described as

$$
\begin{aligned}
& \text { Step 1: } \phi_{\frac{1}{3}}=\left\{\begin{array}{c}
\phi_{0} \ominus k \mathcal{B}_{d} \text { if } v>0 \\
\phi_{0} \oplus k \mathcal{B}_{d} \text { if } v<0
\end{array}\right. \\
& \text { Step 2: } \phi_{\frac{2}{3}}=\left\{\begin{array}{c}
\phi_{\frac{1}{3}} \text { if } \lambda_{2}\left|\nabla \phi_{\frac{1}{3}}\right|\left(I-c_{2}\right)^{2}=\lambda_{1}\left|\nabla \phi_{\frac{1}{3}}\right|\left(I-c_{1}\right)^{2} \\
1 \text { if } \lambda_{2}\left|\nabla \phi_{\frac{1}{3}}\right|\left(I-c_{2}\right)^{2}>\lambda_{1}\left|\nabla \phi_{\frac{1}{3}}\right|\left(I-c_{1}\right)^{2} \\
0 \text { if } \lambda_{2}\left|\nabla \phi_{\frac{1}{3}}\right|\left(I-c_{2}\right)^{2}<\lambda_{1}\left|\nabla \phi_{\frac{1}{3}}\right|\left(I-c_{1}\right)^{2}
\end{array}\right. \\
& \text { Step 3: } \phi=\left(\phi_{\frac{2}{3}} \circ \mathcal{B}_{d}\right) \bullet \mathcal{B}_{d} .
\end{aligned}
$$

which is the of a morphological implementation of a multiphase Active Contour without Edges.

\section{IMPLEMENTATION SPECIFICS}

The implementation of equation (11) is fairly straightforward; however a few comments about the approximation calculations are worth discussing. Foremost, $\phi$ is stored as a binary function. The morphological erosions, dilations, closings, and openings are defined as binary operations in this application. It would be trivial to extend the operations to grayscale values, less trivial for an extension into color or multispectral images. $|\nabla \phi|$ is approximated by the magnitude of the gradient, namely $\sqrt{\phi_{x}{ }^{2}+\phi_{y}{ }^{2}}$ where $\phi_{x}$ and $\phi_{y}$ are computed using finite differences. The Matlab code used to approximate $|\nabla \phi|$ is

$$
\begin{aligned}
& \% \text {--Calculate gradient of } \mathrm{u} \\
& {[\mathrm{m}, \mathrm{n}]=\operatorname{size}(\mathrm{u}) \text {; }} \\
& \mathrm{P}=\operatorname{padarray}\left(\mathrm{u},[1,1], 1, \text { pre' }^{\prime}\right) \text {; } \\
& \mathrm{P}=\operatorname{padarray}\left(\mathrm{P},[1,1], 1, \text { post' }^{\prime}\right) \text {; } \\
& \text { fy }=\mathrm{P}(3: \text { end }, 2: \mathrm{n}+1)-\mathrm{P}(1: \mathrm{m}, 2: \mathrm{n}+1) \text {; } \\
& \mathrm{fx}=\mathrm{P}(2: \mathrm{m}+1,3: \mathrm{end})-\mathrm{P}(2: \mathrm{m}+1,1: \mathrm{n}) \text {; } \\
& \mathrm{G}=\left(\mathrm{fx} . .^{\wedge} 2+\mathrm{fy} .{ }^{\wedge} 2\right) .^{\wedge}(0.5) ; \% \text { magnitude of gradient } \\
& \% \text {--end calculation of gradient of } u \text {. }
\end{aligned}
$$

Additionally, the mean curvature operator, $\left(\phi_{k} \circ \mathcal{B}_{d}\right) \bullet \mathcal{B}_{d}$, can also be expressed as $\left(\phi_{k} \bullet \mathcal{B}_{d}\right) \circ \mathcal{B}_{d}$ for the evolution of $\phi$. To help equalize the influence of both expressions, the implementation of the operators alternate $\left(\phi_{k} \circ \mathcal{B}_{d}\right) \bullet \mathcal{B}_{d}$ and $\left(\phi_{k} \bullet \mathcal{B}_{d}\right) \circ \mathcal{B}_{d}$ throughout the iterations of the main loop. Finally, the choice of the structuring element for our implementation is a square structuring element of size three. In discrete form, the structuring element would take the shape of 


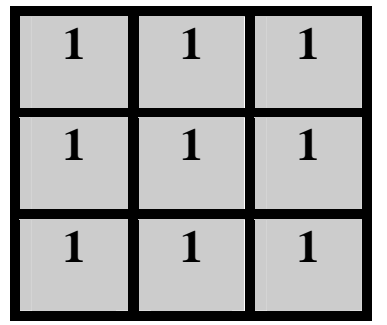

Figure 2: A square structuring element of size 3.

The selection of this structuring element came from the need to discretize the unit ball structuring element used in continuous morphology. Discretizing the morphology is equivalent to discretizing its structuring element, therefore the square structuring element provided a unit area discretization for a three-by-three window in our curve evolution.

In the following section, we will compare the results of the morphological multiphase active contour with the numerical solution to the multiphase Active Contours without Edges contour. In the partial differential equation methods, the algorithms deteriorate the level set function to the point where it is no longer a signed difference function. To fix this shortcoming, it is common to reinitialize the level-set. In the traditional numerical implementation, we chose to use

$$
\phi_{k}=\phi_{k-1} / \sqrt{\phi_{k-1}^{2}+\varepsilon^{2}}
$$

after each iteration of the numerical solution to "re-sign" the level set function. Our morphological multiphase contour did not require a re-initialization step.

\section{EXPERIMENTAL RESULTS}

The images used in the experiments were gathered from the Laboratory of Biomedical Imaging datasets [33]. The images were not contrast enhanced and were acquired with a $50^{\circ}$ fundus camera and digitized with a scanner at 1100x1300 pixels. The authors chose to use the images of vessels pre-processed by a normalization algorithm [34] in a TIF non-compressed color format. Each image was segmented by both algorithms - morphological and traditional - andtwo otherestablished algorithms - the morphological watershed method and the fuzzy c-means - and results in the efficiency and quality of the segmentations were compiled. The results of the experiments underscore the advantages in terms of computational resources, simplicity, and robustness of the morphological algorithm.

\subsection{Morphological and Numerical Active Contours without Edges Experiments}

The parameters used in this part of the experiment are the same for both algorithms: $v=0, \lambda_{1}=$ $\lambda_{2}=1$. Each experimental trial is timed for completion and iterations are recorded.

The first image we use (Arteria-10.tif in the dataset) in our comparison study is an image with a delineated, central vessel. Figure 3 details the original image and the result of segmentation of the image with each algorithm after 300 iterations of each. The morphological algorithm quickly completes the segmentation in 3.1 seconds while the traditional, numerical solution does not finish the segmentation in the given iterations and takes 9.3 seconds to perform the 300 iterations. Due to the strong demarcation of main vessels and surrounding tissue, this particular image does not present a difficult challenge in the region statistics (competition) part of either formulation. 


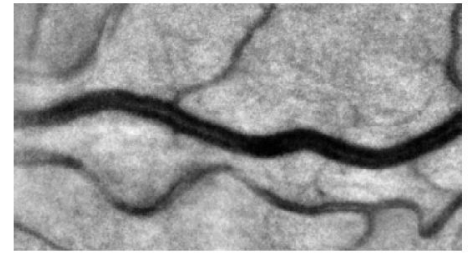

(a)

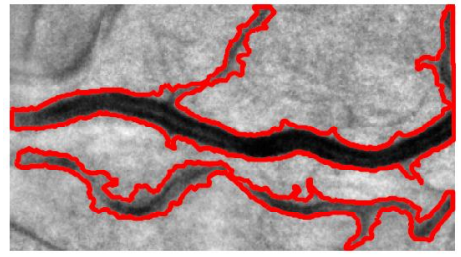

(b)

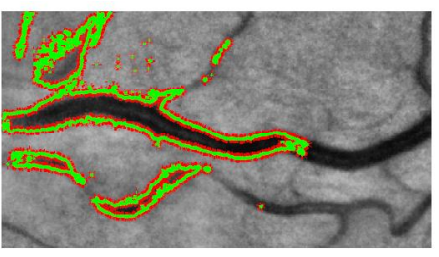

(c)

Figure 3: (a) initial grayscale image, (b) result of Morphological multiphase active contour after 300 iterations (c) result of traditional multiphase Active Contour without Edges after 300 iterations.

The following experiment is conducted on the Arteria-209 image in the dataset. This image yields a faint outline of the vessels and has more intensity homogeneity throughout the image. As a result, the traditional algorithm does not fare well in the segmentation test. The morphological segmentation takes 676 iterations to completely segment the vessel in the image and completes the segmentation in 7 seconds. The multiphase numerical solution takes 31 seconds to achieve 700 iterations and does not successfully segment the image in those iterations. Figure 4 displays the results of this experiment.

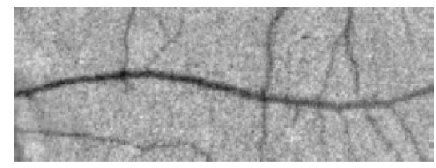

(a)

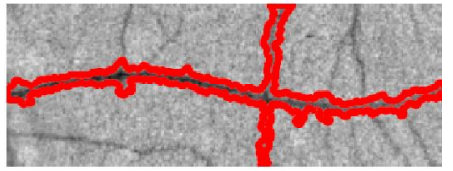

(b)

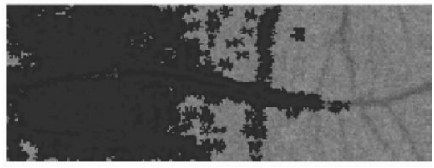

(c)

Figure 4: (a) initial grayscale image, (b) result of Morphological multiphase active contour after 676 iterations, (c) result of traditional multiphase Active Contour without Edges after 700 iterations.

The final exhibition of the success of the morphological segmentation over the numerical solution of the Active Contours without edges can be seen in Figure 5. In this experiment, we use the image Arteria-248. This image is chosen as a challenge image due to its intensity homogeneity, complexity in terms of vessels to detect, and the inhomogeneity of the vessels in the image. The morphological active contour completes the segmentation in 7.8 seconds with 712 iterations. The Active Contours without Edges algorithm takes1092 iterations and 79.4 seconds to achieve a partial segmentation.

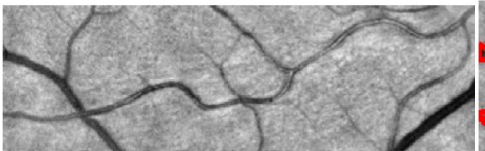

(a)

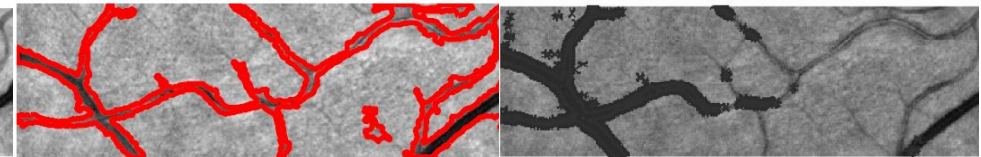

(b)

(c)

Figure 5: (a) initial grayscale image, (b) result of Morphological multiphase active contour after 712 iterations, (c) result of traditional multiphase Active Contour without Edges after 1092 iterations.

\subsection{Morphological Active Contours with Edges and Watershed Experiments}

An established and respected method of segmentation in the medical imaging field, morphological watershed segmentation is a benchmark of comparison for new methods. The efficiency of the watershed method is one of its attractive attributes and since it is almost entirely of morphological operations, it will provide an accurate comparison of efficiency and robustness of the proposed method.

In watershed segmentation, a gray-level image viewed as a topological relief in which the gray level of a given pixel is interpreted as its height. The flooding method of watershed segmentation consists in placing a water source in each regional minimum, flooding the relief from sources, and building barriers when different sources meet [35]. The resulting barriers constitute the 
segmentation of the image. Using Matlab functionality, we segmented the same three images Arteria-10.tif, Arteria-209.tif, and Arteria-248.tif - with the watershed method and compared segmentation results to the morphological multiphase active contour results.

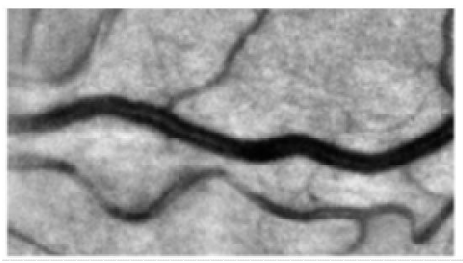

(a1)

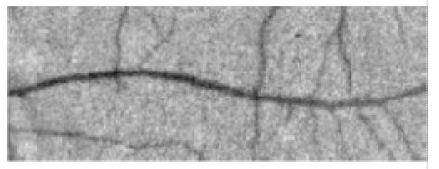

(a2)

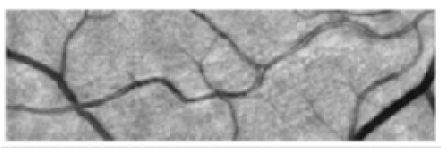

(a3)

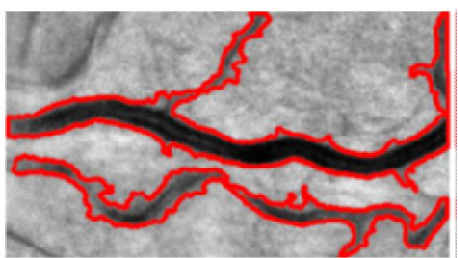

(b1)

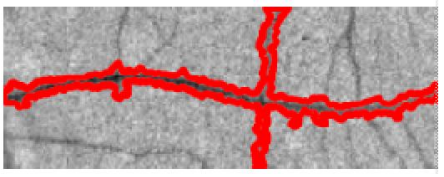

(b2)

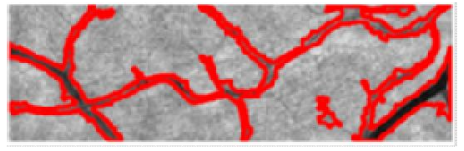

(b3)

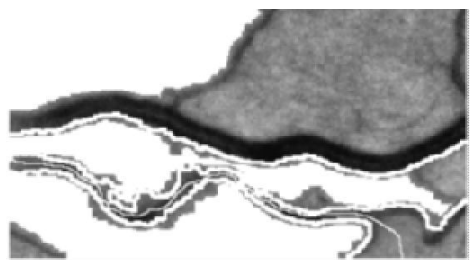

(c1)

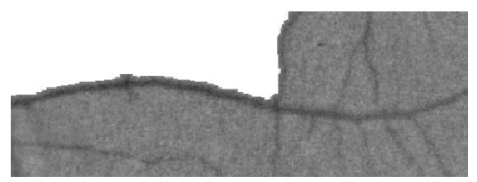

(c2)

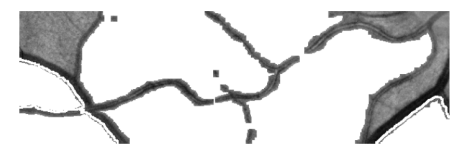

(c3)

Figure 6:( a1), (a2), and( a3) are original input images, (b1), (b2), (b3) are the segmentation results of the morphological multiphase active contour,(c1),(c2),(c3) are the segmentation results of the morphological watershed method.

Since the watershed method is a benchmark method of medical segmentation, the comparison of the segmentations of the two methods clearly shows the morphological active contour method segments vessel images more far more accurately than the watershed method. In efficiency, while the morphological watershed is, on average, faster than the morphological active contour, its lack of accuracy causes the efficiency of the algorithm to be of little significance.

\subsection{Morphological Active Contours without Edges and Fuzzy c-Means Experiments}

Clustering is the process of dividing data elements into classes or clusters so that items in the same class are as similar as possible and items in different classes are as dissimilar as possible. In fuzzy clustering, however, membership values are assigned to pixels that determine the degree to which each pixel belongs to similar and dissimilar classes. Therefore, the measures of similarity and dissimilarity drive fuzzy clustering algorithms. Using this reasoning, a pixel on the edge of the region of interest will have a lower degree of similarity to the cluster than a pixel located near the center of the region of interest. The flexibility inherent in the method in makes fuzzy clustering a natural for the segmentation of complex medical images [35]. In Fuzzy c-means segmentation, the iterative clustering method produces an optimal c partition by minimizing the degree of membership within the group sum of a squared error objective function. The algorithm, traditionally, performs well with low-noise images and any inhomogeneity of object intensity levels does not affect its results. Due to the nature of the images we are using, the fuzzy c-means algorithm is an appropriate algorithm to compare our proposed method.In our experiments, we used a stock fuzzy c-means algorithm to segment the three trial images and then compare the segmentation to the results of the morphological active contour method. 


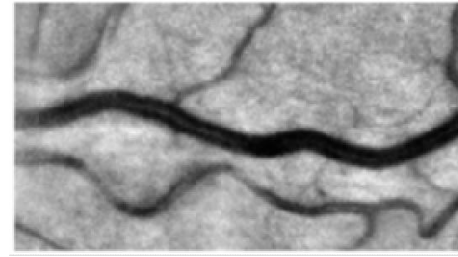

(a1)

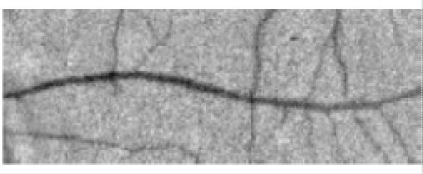

(a2)

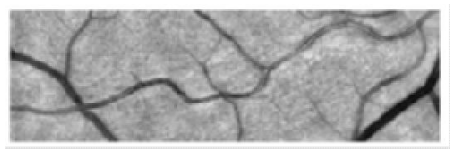

(a3)

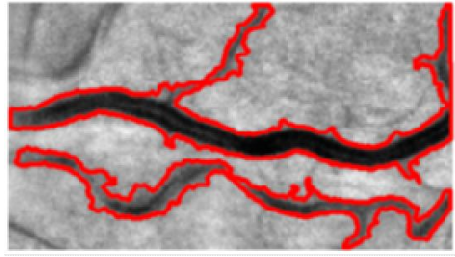

(b1)

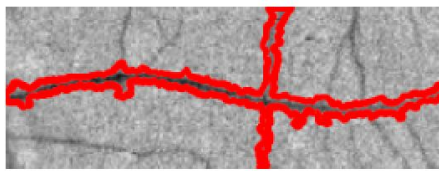

(b2)

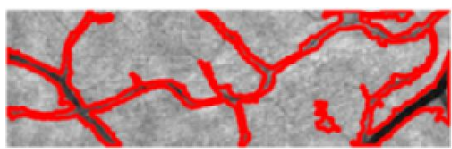

(b3)

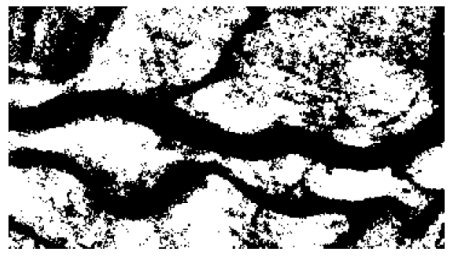

(c1)

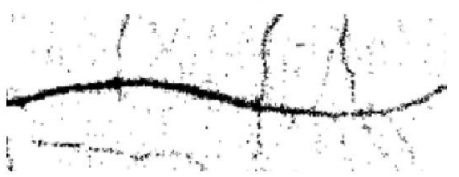

(c2)

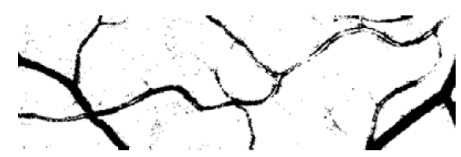

(c3)

Figure 7: (a1), (a2), and( a3) are original input images, (b1), (b2), (b3) are the segmentation results of the morphological multiphase active contour,(c1),(c2),(c3) are the segmentation results of the fuzzy c-means algorithm.

The performance of the fuzzy c-means algorithm is the closest in accuracy to our morphological active contour. However, there are inaccurate sections in the fuzzy c-means segmentation. The first image resulted in over segmentation while the noise/lack of inhomogeneity in the second image resulted in the method over segmenting as well. The third image, chosen for its complexity and variety of intensity levels, provides the optimal situation for a fuzzy c-means algorithm to perform. However, in comparison to our method it did not perform as well and identified objects not belonging to the desired regions of interest as foreground objects. In efficiency, the fuzzy c-means completes the three segmentations in 33 iterations in 4 seconds for Arteria-10, 41 iterations and 4.5 seconds for Arteria-209, and in 46 iterations in 8.1 seconds for Arteria-248. While the times are comparable to our proposed methods, all but the third image do not approach the accuracy garnered from using the morphological active contour scheme. Table 1 summarizes the results of all of the experiments involving the three trial images.

Table 1: Experimental Results

\begin{tabular}{|c|c|c|c|c|c|c|c|c|c|}
\hline \multirow{2}{*}{ Method } & \multicolumn{3}{|c|}{ Arteria-10 } & \multicolumn{3}{|c|}{ Arteria-209 } & \multicolumn{3}{|c|}{ Arteria-248 } \\
\hline & Iterations & Time & Segmented & Iterations & Time & Segmented & Iteration & Time & Segmented \\
\hline $\begin{array}{l}\text { Morphological } \\
\text { active contour }\end{array}$ & 300 & $3.1 \mathrm{~s}$ & yes & 676 & $7 \mathrm{~s}$ & yes & 712 & $7.8 \mathrm{~s}$ & yes \\
\hline $\begin{array}{c}\text { Active } \\
\text { Contour } \\
\text { without Edges }\end{array}$ & 300 & $9.3 \mathrm{~s}$ & no & 700 & $31 \mathrm{~s}$ & no & 1092 & $\begin{array}{c}79.4 \\
\mathrm{~s}\end{array}$ & no \\
\hline Watershed & $\mathrm{n} / \mathrm{a}$ & $3 \mathrm{~s}$ & no & $\mathrm{n} / \mathrm{a}$ & $3 \mathrm{~s}$ & no & $\mathrm{n} / \mathrm{a}$ & $3 \mathrm{~s}$ & no \\
\hline $\begin{array}{c}\text { Fuzzy c- } \\
\text { Means }\end{array}$ & 33 & $4 \mathrm{~s}$ & no & 41 & $4.5 \mathrm{~s}$ & $\begin{array}{c}\text { Over } \\
\text { segmented }\end{array}$ & 46 & $8 \mathrm{~s}$ & $\begin{array}{c}\text { Over } \\
\text { segmented }\end{array}$ \\
\hline
\end{tabular}


International Journal on Bioinformatics \& Biosciences (IJBB) Vol.3, No.3, September 2013

\section{CONCLUSIONS}

This paper introduces a new morphological multiphase active contour model. Based on the multiphase implementation of the Chan-Vese Active Contour without Edges, the morphological active contour efficiently and robustly segmented the trial vascular images. Additionally, it outperformed three of the more commonly used segmentation routines in medical imaging: Active Contour without Edges, morphological watershed, and Fuzzy c-means. The implementation of the morphological multiphase active contour is simpler and has fewer parameters than the numerical version. Additionally, there are no instability issues and no need to re-initialize the level set.

The conducted experiments with the trial vascular images confirm the solutions obtained with the morphological active contour are more accurate in two of the numerical schemes and comparable to the results obtained with fuzzy c-means in one of the trial images. In efficiency, morphological active contours outperform the traditional functional gradient descent counterparts in terms of stability, robustness, and speed. In comparison to the watershed method, the lack of accuracy in the morphological watershed rendered its speed and stability null in comparison to the morphological active contour. The fuzzy c-means algorithm performed more efficiently, but less accurately than the proposed method.

The experiments we have conducted are very promising. The use of the Heaviside function in the image competition portion of the functional lead to more flexibility in the implementation while the structuring element provided an efficient, simple means to approximate mean curvature. We obtained good segmentation results in a variety of vascular images. In future work, the algorithm will be extended to other types of images and to multispectral/color applications.

\section{ACKNOWLEDGEMENTS}

The authors would like to thank Dr. S. Piermarocchi, from the Department of Ophthamology, University of Padova, Italy, for kindly providing the fundas images and the manual tortuosity grading. Additonally, the authors would like to thank the Laboratory of Biomedical Imaging, University of Padova, Italy, for posting the dataset on their webpage: http://bioimlab.dei.unipd.it/Retinal\%20Vessel\%20Tortuosity.htm .

\section{REFERENCES}

[1] Chu, Gregory H., et al. "Automated segmentation of tumors on bone scans using anatomy-specific thresholding." SPIE Medical Imaging.International Society for Optics and Photonics, 2012.

[2] Oke, O. A., et al. "Fuzzy kc-means Clustering Algorithm for Medical Image Segmentation." Journal of Information Engineering and Applications 2.6 (2012): 21-32.

[3] Lin, Phen-Lan, Po-Ying Huang, and Po-Whei Huang. "An automatic lesion detection method for dental x-ray images by segmentation using variational level set." ICMLC. 2012.

[4] Prieto, Juan-Carlos, et al. "CreaLungs: a variational region growing method to segment pulmonary vascular trees." sign 1 (2012): 2.

[5] Valvi, Piyush, et al. "Improved fuzzy c-mean algorithm for Medical Image segmentation." International Journal of Engineering 1.3 (2012).

[6] V. Caselles, F. Catte, T. Coll, and F. Dibos, "A geometric model for active contours in image processing," Numerical Math, 66(1):1-31, 1993.

[7] H. Tek and B. Kimia, "Image segmentation by reaction diffusion in bubbles," in Proceedings of International Conference on Computer Vision, 1995, pp. 156-162.

[8] S. Osher and J. Sethian, "Fronts propagating with curvature-dependent speed: Algorithms based on hamilton-jacobi formulation," Journal of Computational Physics, 79:12-49, 1988. 
International Journal on Bioinformatics \& Biosciences (IJBB) Vol.3, No.3, September 2013

[9] V. Caselles, R. Kimmel, and G. Sapiro, "Geodesic active contours," International Journal of Computer Vision, 22(1): 61-79, 1997.

[10] R. Malladi, J. Sethian, and B. Vemuri, "Shape modeling with front propagation: A level set approach," IEEE Transactions on Pattern Analysis and Machine Intelligence, 17(1):158-175, February 1995.

[11] A. Yezzi, S. Kichenassamy, A. Kumar, et. al, "A geometric snake model for segmentation of medical imagery," IEEE Transactions on Medical Imaging, 16(2):199-209, 1997.

[12] L. Cohen and R. Kimmel, "Global minimum for active contour models: A minimal path approach," International Journal on Computer Vision, 24(1):57-78, 1997.

[13] L. Cohen, "Multiple contour finding and perceptual grouping using minimal paths," Journal of Mathematical Imaging and Vision, 14(3): 225-236, May 2001.

[14] S. Gunn and M. Nixon, "A model based dual active contour," in Proceedings of British Vision Conference, 1994, pp. 305-314.

[15] S. Gunn and M. Nixon, "A robust snake implementation: A dual active contour," IEEE Transactions on Pattern Analysis and Machine Intelligence, 19(1):63-68, January 1997.

[16] G. Aboutanos, J. Nikanne, N. Watkins, and B. Dawant, "Model creation and deformation for the automatic segmentation of the brain in MR images," IEEE Transactions on Biomedical Engineering, 46(11):1346-1356, 1999.

[17] G. Giraldi, L. Goncalves, and A. Oliveira, "Dual topologically adaptable snakes," in Proceedings of International Conference on Computer Vision, Pattern Recognition, and Image Processing, February 2000, pp. 103-107.

[18] M. Dawood, X. Jiang, and K. Schafers, "Reliable dual-band based contour detection: A double dynamic programming based approach,' Lecture Notes in Computer Science, 3212:544-551, 2004.

[19] G. Georgoulas, G. Nikolakopoulos, Y. Koutroulis, et. al, "An intelligent visual-based system for object inspection and welding, relying on active contour models-algorithms," in Proceedings of Second Hellenic Conference on Artificial Intelligence, April 2002, pp. 399-410.

[20] Freixenet, Jordi, et al. "Yet another survey on image segmentation: Region and boundary information integration." Computer Vision-ECCV 2002.Springer Berlin Heidelberg, 2002. 408-422.

[21] T. Chan and L. Vese, "Active contours without edges," IEEE Transactions on Image Processing, 10(2):266-277, 2001.

[22] F. Gibou and R. Fedkiw, "A fast hybrid k-means level set algorithm for segmentation," in $4^{\text {th }}$ Annual Hawaii International Conference on Statistics and Mathematics, November 2005, pp. 281291.

[23] A. Yezzi, A. Tsai, and A. Willsky, “A fully global approach to image segmentation via coupled curve evolution equations," Journal of Visual Communication and Image Representation, 13(1):195-216, March 2002.

[24] Welk, Martin, Michael Breuß, and Oliver Vogel. "Morphological amoebas are self-snakes." Journal of Mathematical Imaging and Vision 39.2 (2011): 87-99.

[25] Marquez-Neila, Pablo, Baumela, Luis, Alvarez, Luis. "A Morphological Approach to Curvaturebased Evolution of Curves and Surfaces." IEEE Transactions on Pattern Analysis and Machine Intelligence, http://doi.ieeecomputersociety.org/10.1109/TPAMI.2013.106, June 2013.

[26] Jalba, Andrei C., and Jos BTM Roerdink. "An efficient morphological active surface model for volumetric image segmentation."Mathematical Morphology and Its Application to Signal and Image Processing.Springer Berlin Heidelberg, 2009.193-204.

[27] Álvarez, Luis, et al. "Morphological snakes." Computer Vision and Pattern Recognition (CVPR), 2010 IEEE Conference on.IEEE, 2010.

[28] Catté, Francine, Françoise Dibos, and Georges Koepfler. "A morphological scheme for mean curvature motion and applications to anisotropic diffusion and motion of level sets."SIAM Journal on Numerical Analysis 32.6 (1995): 1895-1909.

[29] Cao, Frédéric. Geometric curve evolution and image processing.No. 1805.Springer, 2003.

[30] Maragos, Petros, and R. W. Schafer. "Morphological filters--Part II: Their relations to median, order-statistic, and stack filters." Acoustics, Speech and Signal Processing, IEEE Transactions on 35.8 (1987): 1170-1184.

[31] Alvarez, Luis, et al. "Axioms and fundamental equations of image processing." Archive for rational mechanics and analysis 123.3 (1993): 199-257. 
International Journal on Bioinformatics \& Biosciences (IJBB) Vol.3, No.3, September 2013

[32]

Zhao, Hong-Kai, et al. "A variational level set approach to multiphase motion." Journal of computational physics 127.1 (1996): 179-195.

[33] Grisan, Enrico, Marco Foracchia, and Alfredo Ruggeri. "A novel method for the automatic grading of retinal vessel tortuosity."Medical Imaging, IEEE Transactions on 27.3 (2008): 310319.

[34] Foracchia, Marco, Enrico Grisan, and Alfredo Ruggeri. "Luminosity and contrast normalization in retinal images." Medical Image Analysis 9.3 (2005): 179-190.

[35] Noor, N. M., et al. "Comparing watershed and FCM segmentation in detecting reticular pattern for interstitial lung disease." Biomedical Engineering and Sciences (IECBES), 2012 IEEE EMBS Conference on.IEEE, 2012.

\section{Authors}

Currently a graduate student in Computational Science at University of Arkansas at Little Rock, Victoria L. Fox is also a mathematics instructor for the University of Arkansas at Monticello. Her professional interests include morphological image processing, segmentation of natural images, and the incorporation of fuzzy logic in multispectral image segmentation.

MariofonnaMilanova is a Professor of Computer Science Department at the University of Arkansas at Little Rock since 2001. She received her M. Sc. degree in Expert Systems and AI in 1991 and her Ph.D. degree in Computer Science in 1995 from the Technical University, Sofia, Bulgaria. Dr.Milanova did her post-doctoral research in visual perception at the University of Paderborn, Germany. She has extensive academic experience at various academic and research organizations in different countries. Milanova serves as a book editor of two books and associate editor of several international journals. Her main research interests are in the areas of artificial intelligence, biomedical signal processing and computational neuroscience, computer vision and communications, machine learning, and privacy and security based on biometric research. She has published and co-authored more than 70 publications, over 43 journal papers, 7 book chapters, numerous conference papers and 2 patents.

Salim Al-Ali is a Ph.D. graduate student in the integrated computing of computer science department at University of Arkansas at Little Rock (UALR). He received a master degree from computer science department, Baghdad University, Iraq on 1995. He is working as a teacher in Dohuk Technical Institute at Dohuk Polytechnic University. His research interest field is computer vision in general, human action recognition, image and video understanding.
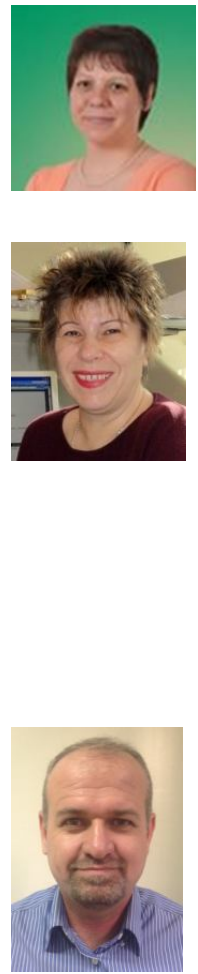